\title{
Large polycrystalline optical germanium Ge:Na plates with improved optical parameters and their application
}

\author{
G.S. Pekar ${ }^{1}$, A.F. Singaevsky ${ }^{1}$, M.M. Lokshin' ${ }^{1}$, V.I. Gordienko², I.V. Mazurin ${ }^{2}$ \\ ${ }^{1}$ V. Lashkaryov Institute of Semiconductor Physics, NAS of Ukraine, \\ 41, prospect Nauky, 03680 Kyiv, Ukraine, \\ E-mail:pekar@isp.kiev.ua,511alexsin@gmail.com \\ ${ }^{2}$ State Enterprise Research \& Production Complex "Photoprylad", \\ 85, B. Vyshnevetskoho str., 18001 Cherkasy, Ukraine
}

\begin{abstract}
It has been experimentally shown that transmission and scattering of IR radiation by Na-doped coarse-grained large germanium plates are the same as of Ge:Na single crystals and exceeds the parameters in the commonly used optical germanium Ge:Sb grown from the same raw material. Being based on experimental results, a conclusion has been made that the nature of the dopant in $\mathrm{Ge}: \mathrm{Na}$ is a decisive factor defining its optical parameters, along with the usual requirements of high purity of the raw material, resistance values below about $20 \mathrm{Ohm} \cdot \mathrm{cm}$ and a sufficiently large grain size (for polycrystalline material). It is assumed that, most likely, $\mathrm{Na}$ in $\mathrm{Ge}: \mathrm{Na}$, contrary to $\mathrm{Sb}$ in $\mathrm{Ge}: \mathrm{Sb}$, doesn't form impurity clouds that scatter the incident IR radiation. The advantages of the polycrystalline Ge:Na as a material for IR optics were proved when using it for industrial manufacturing the protective screens for night vision systems.
\end{abstract}

Keywords: Na-doped germanium, large coarse-grained plates, optical transmission, protective screens, thermal imaging systems.

doi: https://doi.org/10.15407/spqeo21.02.173

PACS 07.05.Bx, 42.30.-d, 61.72.Ff

Manuscript received 18.06.18; revised version received 14.06.18; accepted for publication 27.06.18; published online 03.07.18.

\section{Introduction}

Optical germanium that is transparent to infrared (IR) radiation in the regions of both atmospheric transparency windows is one of the basic materials for IR optics. For more than half a century, the mono- and polycrystalline optical germanium is used to manufacture lenses, windows, and other passive elements of IR technique. Recently, a special attention has been paid to polycrystalline optical germanium that is cheaper and, what is important, may be more easily grown in the form of large-area plates required, in particular, for production of protective screens for thermal imaging systems of night vision.

To ensure that the replacement of single crystals with polycrystalline material does not impair the parameters of Ge-based devices, it is necessary to know to what extent different characteristics, and primarily the optical parameters, of polycrystalline optical germanium are inferior to the characteristics of single crystals and whether it is possible to make these characteristics as close as possible. The technological and physical experiments described in this paper are aimed at solving this problem. It has been shown that, by using a properly selected doping of optical germanium, namely, doping with an interstitial donor impurity $(\mathrm{Na})$, but not with a commonly used substitutional donor impurity (such as $\mathrm{Sb}$ ), it is possible to grow large polycrystalline plates, which optical parameters are the same as in the goodquality single crystals of optical germanium grown from the same raw material.

\section{Related works}

As it was shown about half a century ago [1], to provide a high transparency of $\mathrm{Ge}$ crystals in the IR region, doping with donor-type impurities in a definite range of their concentrations has to be used. This doping has to ensure the $n$-type conductivity of germanium, which makes it possible to avoid, upon absorption of IR radiation, direct transitions of carriers between the subbands of the valence band. Just these direct transitions lead to high absorption of IR radiation in Ge. However, the level of Ge doping with donor impurities must be not too high to avoid increased absorption by free electrons. 
It was concluded that the density of donors which provides the crystal resistivity from 5 to $40 \mathrm{Ohm} \cdot \mathrm{cm}$ (the respective values of free electron concentrations lies approximately between $4 \cdot 10^{14}$ and $5 \cdot 10^{13} \mathrm{~cm}^{-3}$ [2]) is optimal for preparing the optical germanium for applications in IR technique. In fact, the best optical parameters were observed experimentally in somewhat narrower resistivity ranges. According to our experiments, this region lies between 10 and $25 \mathrm{Ohm} \cdot \mathrm{cm}$ at room temperature, which roughly coincides with some data published previously [3, 4].

The donor impurity that is most often used and provides the necessary optical properties of Ge crystals is antimony, atoms of which substitute Ge atoms in the crystal lattice. However, we have managed to dope Ge crystals with an interstitial $\mathrm{Na}$ and to show that the optical parameters of single-crystalline and coarsegrained $\mathrm{Ge}: \mathrm{Na}$ are better as compared with those of Ge:Sb grown from the same raw material $[5,6]$.

In recent years, in connection with the requirements of industry to reduce the cost and at the same time to increase the size of optical germanium crystals that can be provided by using an available polycrystalline material, the issues related to the differences in the optical characteristics of single-crystalline and polycrystalline optical germanium have become rather topical [7-13].

A detailed critical comparison of the characteristics of single-crystalline and polycrystalline optical germanium has been carried out by Schroeder and Rosolowski in 2013 [7]. In particular, the well-known fact has been analyzed that, for the wavelength of $10.6 \mu \mathrm{m}$, the values of the manufacturer's guaranteed transmission of the 1-cm-thick single-crystalline and polycrystalline windows are 0.020 and $0.035 \mathrm{~cm}^{-1}$, respectively. It means that the transmission of $10.6-\mu \mathrm{m}$ radiation by uncoated crystals of both types are 45.86 and $44.99 \%$, respectively, and in the case of using a perfect antireflection coating, transmission equals 98.02 and $96.56 \%$, respectively. Thus, the difference in transmission of the single-crystalline and polycrystalline germanium with an antireflection coating exceeds $1.4 \%$. On the assumption of these data, as well as the data on the differences in the index homogeneity, the authors came to the conclusions that (i) the use of Ge single crystals is desirable, if $1 \%$ improvement in optical transmittance is crucial, for example, for systems containing multiple Ge lenses in series, and (ii) polycrystalline germanium is more suitable for creation of optical elements with small to medium sizes.

At the same time, some authors had more optimistic view on the use of polycrystalline germanium. In particular, Adams [8] concluded that, since, on the one hand, the polycrystalline blanks may consist of large crystallites and, hence, may have few grain boundaries, but, on the other hand, the single crystals may contain many dislocations, many small angle boundaries and many slip planes, from the viewpoint of optical elements manufacturing, the Ge blanks classification as singlecrystalline and polycrystalline is an oversimplification.
The author approves that polycrystalline material in most cases can be used on a par with single crystals, only it must be properly manufactured. In particular, as it was shown by McNatt and Handler [12], polycrystalline germanium should have a sufficiently low resistivity, say from 4 to $25 \mathrm{Ohm} \cdot \mathrm{cm}$. This is necessary in order to compensate acceptors (for example, $\mathrm{Cu}$ and $\mathrm{Au}$ ), which may be accumulated at the grain boundaries, creating regions of increased absorption. Such regions, with resistivity above $40 \mathrm{Ohm} \cdot \mathrm{cm}$, were detected by Lewis et al. [13] in Ge with the resistivity between 20 and $40 \mathrm{Ohm} \cdot \mathrm{cm}$, and the authors have concluded that this effect associated with the grain boundaries can be eliminated by using high-purity starting materials.

Lines 1 and 2 in Table 1 show the values of Ge theoretical maximum transmission and the recommended minimum transmission taken from [8]. The lines 3 to 5 show the experimental values of transmission of $\mathrm{Ge}: \mathrm{Sb}$ and Ge:Na single crystals grown from the same raw material. Those data were first published by us in [14].

Let's make two comments to Table 1. Firstly, sometimes the values of $\mathrm{Ge}$ transmission at different wavelengths given in the literature are higher than even theoretical values shown in the line 1 of Table 1 . Most probably, this is due to the overestimation of the transmission values obtained by those measurements in which scattering and its apparent contribution to the crystal transmission was not taken into account [10]. Secondly, although the values of transmission of $\mathrm{Ge}: \mathrm{Sb}$ crystals shown in the line 3 are very close to the data given by industrial manufacturers of optical germanium, sometimes they can be slightly larger or smaller for some wavelengths. Most likely, this is due to the different chemical contaminations in the raw materials and proves once again that in order to establish the role of dopant in

Table 1. Optical tramsmission of different optical germanium crystals.

\begin{tabular}{|c|c|c|c|c|c|c|c|c|c|c|}
\hline z & Wavelength, $\mu \mathrm{m}$ & 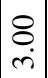 & 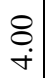 & $\begin{array}{l}8 \\
\dot{1} \\
\end{array}$ & $\mid$\begin{tabular}{l}
8 \\
\hdashline \\
0
\end{tabular} & $\stackrel{8}{\circ}$ & $\underset{\infty}{8}$ & \begin{tabular}{c}
8 \\
\hdashline \\
\hdashline
\end{tabular} & $\begin{array}{l}8 \\
\dot{0} \\
0\end{array}$ & $\stackrel{8}{\circ}$ \\
\hline 1 & $\begin{array}{l}\text { Theoretical maximum } \\
\text { transmission }\end{array}$ & $\begin{array}{l}0 \\
\dot{b} \\
\dot{q}\end{array}$ & $\begin{array}{l}\infty \\
\dot{b} \\
\dot{f}\end{array}$ & $\begin{array}{l}\infty \\
\infty \\
0 \\
\dot{f} \\
\end{array}$ & $\begin{array}{l}n \\
\sigma \\
\dot{0} \\
\gamma\end{array}$ & 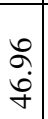 & $\begin{array}{l}\infty \\
\stackrel{2}{0} \\
\dot{b}\end{array}$ & $\begin{array}{c}8 \\
\dot{+} \\
\text { f }\end{array}$ & $\begin{array}{c}8 \\
\dot{+} \\
\dot{\gamma}\end{array}$ & 官 \\
\hline 2 & $\begin{array}{l}\text { Recommended minimum } \\
\text { transmission }\end{array}$ & $\ddot{q}$ & $\vec{q}$ & $\begin{array}{l}\tilde{y} \\
\dot{q} \\
\dot{y}\end{array}$ & $\begin{array}{l}-7 \\
\dot{b}\end{array}$ & $\begin{array}{l}q \\
\dot{\gamma}\end{array}$ & $\stackrel{r}{\sigma}$ & 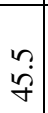 & $\begin{array}{l}m \\
⿱ ⺌ \\
q\end{array}$ & $\stackrel{f}{f}$ \\
\hline 3 & $\begin{array}{l}\text { Transmission of Ge:Sb } \\
\text { single crystal, \% }\end{array}$ & $\begin{array}{l}\stackrel{n}{f} \\
\stackrel{+}{0} \\
\dot{f}\end{array}$ & 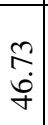 & $\begin{array}{l}\hat{\imath} \\
\dot{\sigma} \\
\dot{q}\end{array}$ & $\mid \begin{array}{l}0 \\
a \\
\check{\gamma} \\
\forall\end{array}$ & $\begin{array}{l}\stackrel{8}{ } \\
\stackrel{+}{f}\end{array}$ & $\begin{array}{l}\stackrel{n}{m} \\
\stackrel{f}{f}\end{array}$ & $\begin{array}{l}5 \\
\dot{0} \\
\dot{y}\end{array}$ & $\begin{array}{l}\stackrel{2}{ } \\
\text { 寸े }\end{array}$ & $\begin{array}{l}\curvearrowright \\
\stackrel{f}{f}\end{array}$ \\
\hline 4 & $\begin{array}{l}\text { Transmission of Ge:Na } \\
\text { single crystal, \% }\end{array}$ & $\vec{\nabla}$ & $\begin{array}{c}\stackrel{v}{r} \\
f \\
\dot{f}\end{array}$ & 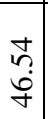 & $\mid \begin{array}{l}\infty \\
\vec{b} \\
\dot{+} \\
+\end{array}$ & $\begin{array}{l}\hat{0} \\
\dot{0} \\
\dot{y}\end{array}$ & 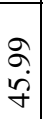 & 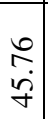 & $\vec{\nabla}$ & $\begin{array}{l}\hat{b} \\
\ddot{f}\end{array}$ \\
\hline 5 & $\begin{array}{l}\text { Relative increase in the } \\
\text { Ge:Na optical } \\
\text { transmission as compared } \\
\text { with Ge:Sb,\% }\end{array}$ & $\stackrel{8}{:}$ & $\stackrel{\sim}{n}$ & $\begin{array}{c}5 \\
0 \\
0\end{array}$ & $\stackrel{\infty}{+\infty}$ & $\stackrel{0}{0}$ & $\stackrel{乛}{\ominus}$ & $\stackrel{n}{n}$ & $\underline{\sigma}$ & $\stackrel{\infty}{\infty}$ \\
\hline
\end{tabular}


formation of material properties, it is necessary to compare the transmission of differently doped crystals grown in the same laboratory and from the same raw material.

As it was shown by us previously [5], among the advantages of Ge:Na crystals over Ge:Sb crystals there is a significantly lower scattering of transmitted radiation. This is due not only to the fact that introduction of smallsize interstitial $\mathrm{Na}$ atoms into germanium leads to less distortions of the crystal lattice as compared with substitutional impurity $\mathrm{Sb}$ in $\mathrm{Ge}$, but also to lower solubility of $\mathrm{Na}$ in $\mathrm{Ge}$ (about $10^{15}$ atoms $/ \mathrm{cm}^{3}$ ) [6] as compared with $\mathrm{Sb}$. The maximum solubility of $\mathrm{Sb}$ in $\mathrm{Ge}$ crystals is $1.2 \cdot 10^{19}$ atoms $/ \mathrm{cm}^{3}$ [15], Sb has a retrograde solubility in $\mathrm{Ge}$, and, as a result, while cooling the grown $\mathrm{Ge}: \mathrm{Sb}$ crystals from the growth temperature to the room one, second-phase inclusions of $\mathrm{Sb}$ with characteristic sizes about $6 \ldots 9 \mu \mathrm{m}$ are formed, and they scatter the infrared radiation [16]. Unlike this, the density of dissolved $\mathrm{Na}$ in $\mathrm{Ge}$ is low, and formation of impurity clouds [17] that serve as scattering centers in $\mathrm{Ge}: \mathrm{Na}$ is likely much less than in Ge:Sb.

\section{Growth of polycrystalline Ge: $\mathrm{Na}$ ingots and plates}

As a starting material for crystal growing, a zone-refined polycrystalline germanium with a purity of at least $6 \mathrm{~N}$ was used. Polycrystalline Ge:Na bulk crystals were grown by two methods.

For manufacturing comparatively small optical germanium windows, polycrystalline $\mathrm{Ge}: \mathrm{Na}$ ingots were pulled from the melt by Czochralski method in the form of rectangular parallelepipeds $88 \times 82 \times 47 \mathrm{~mm}$ in size. The ingots were grown on single-crystalline seeds inside a graphite shaper which presents an empty box with the above-indicated internal dimensions. Growth was carried out in the argon or helium ambient. The size of individual grains in the grown ingots was usually $0.5 \ldots .5 .0 \mathrm{~cm}^{2}$. For practical use, the ingots were cut into windows with an area of $82 \times 47 \mathrm{~mm}^{2}$.

As for manufacturing larger windows or protective screens, the Ge:Na plates up to $400 \times 120 \times 20 \mathrm{~mm}$ in size were grown by a horizontal directed crystallization in a nitrogen ambient. Among the advantages of this method [18] are its relative simplicity, the capability to grow very large plates as well as their cost-effectiveness. The grown plates were polycrystalline with the grain sizes of 1 to $10 \mathrm{~cm}^{2}$. Similarly to $\mathrm{Ge}: \mathrm{Na}$ crystals pulled from the melt [5], the resistance of the plates was fairly uniform along their length, decreasing from the beginning of the plate to its end by no more than by $10-12 \%$, most often approximately from 18 to $16 \mathrm{Ohm} \cdot \mathrm{cm}$. The reasons for this higher homogeneity in Ge: Na crystals as compared, for example, with crystals doped with $\mathrm{V}$ group elements, have been discussed by us previously [6]. Using specially developed technological tricks, the crystallization front was made maximally close to flat, which, in particular, contributed to a reduction of mechanical stresses in the plates [18].

\section{Results and discussion}

\subsection{Optical transmission of Na-doped mono- and polycrystalline germanium}

In Introduction, it was already noted that in the traditional optical germanium $\mathrm{Ge}: \mathrm{Sb}$ the absorption coefficient (more precisely, the coefficient of radiation extinction) in mono- and polycrystals differs by more than one and a half times $\left(0.02\right.$ and $0.035 \mathrm{~cm}^{-1}$, respectively). Before analyzing the properties of monoand polycrystalline Ge:Na, we shall discuss the difference in the optical transmission of $\mathrm{Ge}: \mathrm{Na}$ and $\mathrm{Ge}: \mathrm{Sb}$ single crystals. The doped crystals of both types were grown from the same raw material, as well as by using the same technological equipment and the same growth conditions. Optical transmission of the grown crystals is shown in the lines 3 and 4 of Table 1 . The line 5 of Table shows by how many percent the transmission of $\mathrm{Ge}: \mathrm{Na}$ exceeds the transmission of $\mathrm{Ge}: \mathrm{Sb}$. For example, at the practically important wavelength of the incident radiation $10.6 \mu \mathrm{m}$, the transmission of $\mathrm{Ge}: \mathrm{Na}$ is about $1.75 \%$ higher than that of Ge:Sb crystals.

However, while using optical germanium plates as a material for fabrication of the protective screens, the value of the radiation scattering is especially important to provide an image of good quality. It is known [10] that in $\mathrm{Ge}: \mathrm{Sb}$ crystals the radiation scattering may be rather significant and can even reach $23 \%$. Taking into account that the maximal transmission of IR radiation achieved in optical Ge equals $47 \%$ (without antireflection coating), this means that up to half of the total radiation passing through the crystal can deviate from its original direction. Previously we determined [5] that the values of the radiation scattering and directional transmission of $10.6 \mu \mathrm{m}$ radiation equal $4.0-5.0$ and $39 \%$, respectively, in Ge:Sb and 1.2-1.5 and 41\%, respectively, in Ge:Na. In these experiments, we investigated crystals $\mathrm{Ge}: \mathrm{Sb}$ and Ge:Na grown by us from the same source material, as well as the industrial crystals Ge:Sb. It turned out that the results obtained for $\mathrm{Ge}: \mathrm{Sb}$ crystals of both origins were almost identical.

Fig. 1a shows the optical transmission spectra of two randomly selected windows with the diameter $2.54 \mathrm{~cm}$ and thickness $0.1 \mathrm{~cm}$, cut from the polycrystalline Ge: Na ingot, $88 \times 82 \times 47 \mathrm{~mm}$ in size, grown by us by pulling from the melt in He atmosphere, as described above in the section 2.1. The measurements were carried out at the "Axsys Technologies" company (USA). For comparison, the transmission of singlecrystalline $\mathrm{Ge}: \mathrm{Sb}$ window used a reference one is shown. It can be seen that the transmission of the reference single-crystalline sample exactly coincides with the transmission of one of the $\mathrm{Ge}: \mathrm{Na}$ polycrystalline 

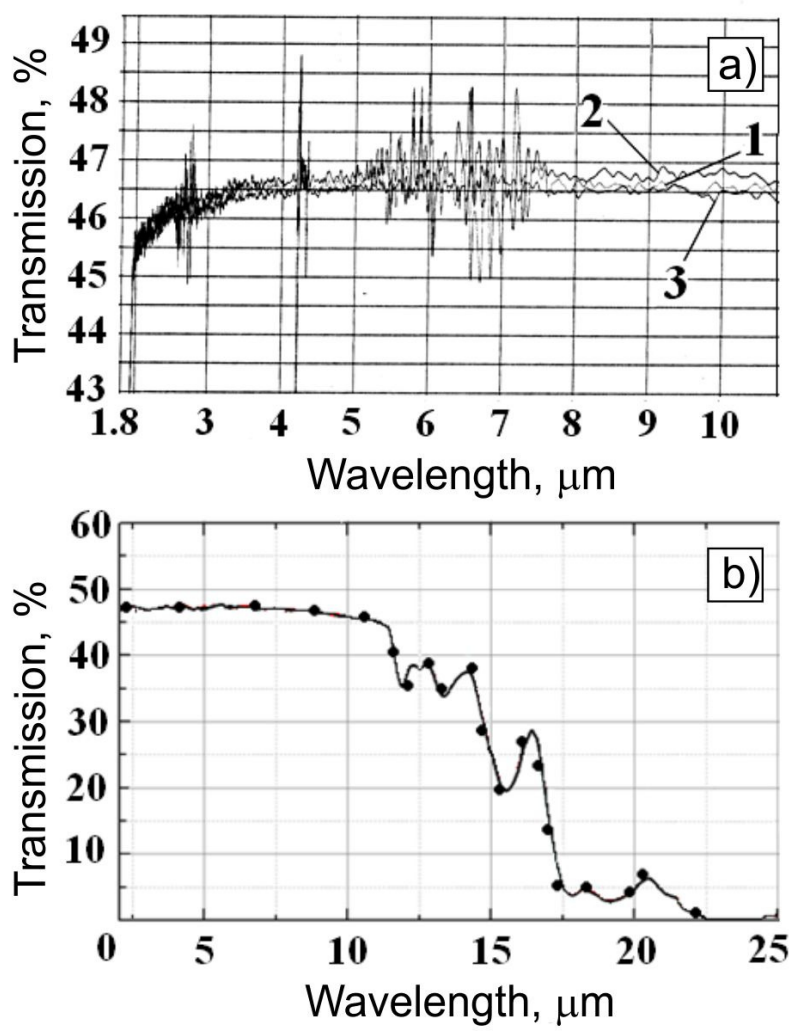

Fig. 1. (a) Optical transmission spectra of two randomly selected windows with the diameter $2.54 \mathrm{~cm}$ and thickness $1 \mathrm{~mm}$, cut from the polycrystalline Ge:Na ingot, $88 \times 82 \times 47 \mathrm{~mm}$ in size, grown by pulling from the melt in He atmosphere. For comparison, the transmission of a high-quality $\mathrm{Ge}: \mathrm{Sb}$ window with the same thickness, used as a reference one, is shown. (b) The optical transmission spectrum of the window with the thickness $5 \mathrm{~mm}$, cut from a Ge:Na coarse-grained plate $330 \times 120 \times 20 \mathrm{~mm}$ in size grown by the method of horizontal directed crystallization in an nitrogen ambient. For comparison, the typical transmission of the Ge:Na single crystal grown from the same raw material is shown (solid circles). The measurements were made at room temperature by using an Infrared Vacuum Fourier Spectrometer Bruker Vertex 70V.

windows and even is slightly smaller than the transmission of the second polycrystalline $\mathrm{Ge}: \mathrm{Na}$ window. Thus, the optical transmission of polycrystalline $\mathrm{Ge}: \mathrm{Na}$ grown by pulling from the melt is at least no less than the transmission of single-crystalline Ge:Sb highquality reference sample.

Fig. 1b shows the optical transmission spectrum of a window with the thickness $5 \mathrm{~mm}$, cut from the $\mathrm{Ge}: \mathrm{Na}$ coarse-grained plate $330 \times 120 \times 20 \mathrm{~mm}$ in size grown by us using the method of horizontal directed crystallization in an nitrogen ambient. For comparison, the typical transmission of the Ge: Na single crystal grown from the same raw material is shown (solid circles). As seen, the transmission spectra of both crystals completely coincide.

The above results allow us to draw important conclusions concerning the optical properties of polycrystalline optical germanium Ge:Na. They show that, in addition to previously established such important technological factors as the reduction of the temperature fluctuations in Ge melt, sustaining the instant crystal growth rates and other improvements of the growing conditions [10], the nature of the dopant also can play a decisive role in improvement of optical parameters of the crystalline optical germanium. As the above results show, replacing the substitutional impurity $\mathrm{Sb}$ with the interstitial impurity $\mathrm{Na}$ not only leads to the improvement of the optical parameters of single crystals without taking special technological efforts, but also the optical characteristics of polycrystalline ingots and polycrystalline large plates, regardless of the method of their growing, are the same as in high-quality single crystals. We emphasize once more that this is most likely due to the absence, in $\mathrm{Ge}: \mathrm{Na}$, of impurity clouds, which serve as scattering centers for IR radiation (see above, Section 2).

\subsection{Practical benefits of using polycrystalline Ge:Na plates}

When manufacturing protective screens based on polycrystalline Ge:Na plates, it turned out that the above advantages of this material are most noticeable at the final stage of screen manufacturing. In particular, it was found that the comparatively small excess of the Ge:Na optical transmission in comparison with Ge:Sb becomes higher by a few percent after applying an anti-reflective coating on both sides of the plate. This is apparently associated with a difference in the nature of the defects that exist at the crystal-coating interface in the both types of materials.

From the viewpoint of the practical use of Ge:Na crystals, it is very important that IR radiation scattering in Ge:Na single and polycrystals is lower than in Ge:Sb crystals grown from the same raw material. As it was mentioned above in Section 3, when $\mathrm{Sb}$ is replaced by $\mathrm{Na}$ as a dopant in $\mathrm{Ge}$, the values of the radiation scattering decrease more than threefold. In the well-known BeerLambert-Bouguer law $I(d)=I_{0} \exp \left(-k_{\lambda} d\right)$, (where $I_{0}$ and $I(d)$ are the intensities of the incident and transmitted radiation, $d$ is the sample thickness), the extinction coefficient $k_{\lambda}$ includes the values of the coefficients that determine the absorption of radiation by the crystal lattice and the scattering of radiation in the sample. Since in the case of replacing $\mathrm{Sb}$ by $\mathrm{Na}$, as a rule, the scattering decreases much more strongly than absorption, it could be expected that, when using Ge:Na protective screens in thermal night vision systems, the optical resolution of the image will be noticeably higher than when using Ge:Sb screens.

Figs. $2 \mathrm{a}$ and $2 \mathrm{~b}$ shows the results of testing the thermal imaging system of night vision using the protective screens made of $\mathrm{Ge}: \mathrm{Sb}$ (a) and $\mathrm{Ge}: \mathrm{Na}$ (b) polycrystalline plates grown from the same raw material. The tests were carried out at night, the night vision system with a Ge:Na protective screen was brought up to such a maximum distance from the object, at which it was already possible to determine its presence and 

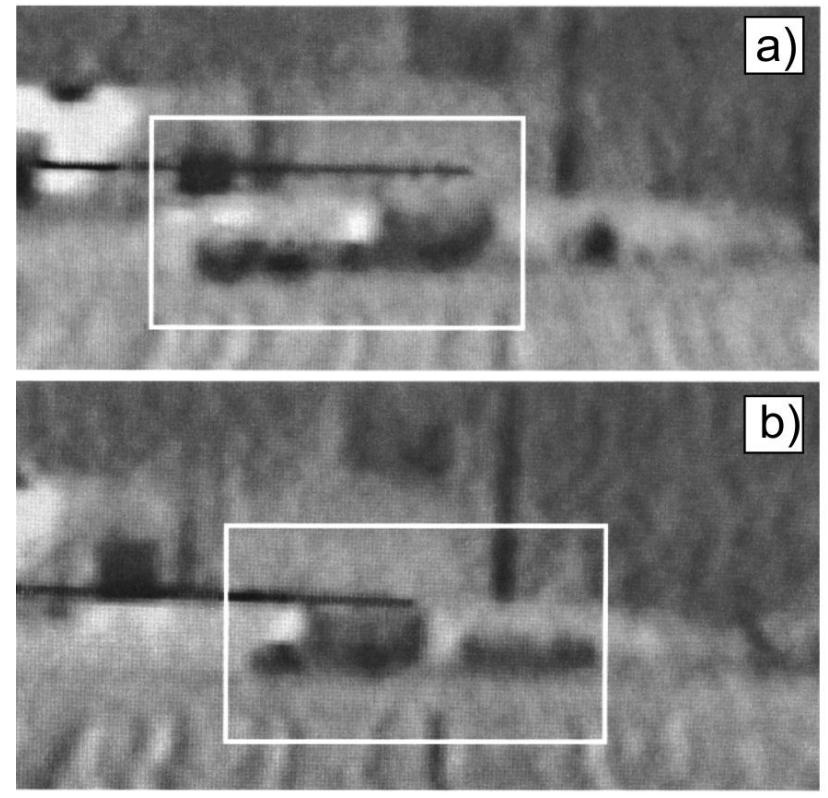

Fig. 2. Results of testing the parameters of the thermal imaging system of night vision using protective screens made of $\mathrm{Ge}: \mathrm{Sb}$ (a) and $\mathrm{Ge}: \mathrm{Na}$ (b) coarse-grained plates grown from the same raw material. The tests were performed at night, the distance to the monitored object (truck) was $3580 \mathrm{~m}$.

nature. At a distance to the monitored object $3580 \mathrm{~m}$ and while using a coarse-grained $\mathrm{Ge}: \mathrm{Na}$ protective screen, the experienced observer confidently determined that the monitored object is seen and it is a truck of the brand "Gazelle" (Fig. 2b). However, after replacing the Ge:Na protective screen with a Ge:Sb protective screen with thesame geometric parameters, the existence of the object could be also detected but its nature could not be distinguished (Fig. 2a).

Taking into account that the photos shown in Fig. 2 may seem not very convincing and someone can doubt the observer's conclusions about those images that he saw with his eyes and in order to provide the quantitative information about the quality of both images shown in Fig. 2, we estimated roughly the degree of blurring for those images. Blurring is usually understood as unclear outlines of the elements of the image, which does not allow to discern details of the image. One of the methods for estimating the degree of image blur is based on the value of the Weibull distribution parameter $\eta$ : the greater the degree of blur, the closer the value of this parameter approaches the value of 2 [19]. According to this criterion, the image in Fig. $2 b$ does not exceed the value of $\eta \approx 1.3 \ldots 1.4$ which is considered quite sufficient to establish the nature of the object, while the image in Fig. 2 a exceeds the value of $\eta \approx 1.7$.

Owing to rather good optical parameters of large polycrystalline Ge:Na plates as well as to reliability and comparative cheapness of their growing, for several recent years this material has already found successful industrial use to create protective screens with dimensions up to $400 \mathrm{~cm}^{2}$ for thermal imaging systems.

\section{Conclusions}

It has been experimentally shown that the practically important optical parameters, such as transmission and scattering of IR radiation, of $\mathrm{Na}$-doped coarse-grained large germanium plates is the same as of Ge: Na single crystals and are better than the parameters of the commonly used optical germanium Ge:Sb grown from the same raw material. The best values of those optical parameters of mono- and polycrystalline $\mathrm{Ge}: \mathrm{Na}$ in comparison with Ge:Sb may be attributed to the absence of impurity clouds in Ge:Na (primarily as a result of the low solubility of $\mathrm{Na}$ in $\mathrm{Ge}$ ) which scatter the incident IR radiation. It is concluded that the nature of the dopant in mono- and polycrystalline optical germanium is a decisive factor determining optical parameters of this material, along with the degree of chemical purity of the raw material. Under conditions that the value of the resistivity in polycrystalline Ge:Na large plates do not exceed approximately $20 \mathrm{Ohm} \cdot \mathrm{cm}$ and the size of the grains is sufficiently large, this material is shown to have important advantages when using it as a material for IR optics, in particular, the best image resolution in thermal imaging systems of night vision. It was proved when using this material in industrial applications.

\section{Acknowledgements}

This work was funded by the National Academy of Sciences of Ukraine as well as by the Ministry of Education and Science of Ukraine via the Project DZ/352017.

\section{References}

1. Pankove J. Optical Processes in Semiconductors. Prentice-Hall, Inc., Englewood Cliffs, New Jersey, 1971.

2. Prince M.B. Drift mobilities in semiconductors. I. Germanium. Phys. Rev. 1953. 92. P. 681-687.

3. Capron E., Brill O. Resistance for Optical Germanium at $10.6 \mu \mathrm{m}$. Appl. Opt. 1973. 12. P. 569-572.

4. Hutchinson C., Lewis C., Savage J., Pitt A. Surface and bulk absorption in germanium at $10.6 \mu \mathrm{m}$. Appl. Opt. 1982. 21. P. 1490-1495.

5. Pekar G.S., Singaevsky A.F. Na-doped optical germanium bulk crystals. Appl. Phys. A. 2012. 108. P. 657-664 (doi: 10.1007/s00339-012-6947-x).

6. Pekar G.S., Singaevsky A.F. Solubility, diffusion and electrical activity of $\mathrm{Na}$ in bulk Ge crystals. Mater. Sci. in Semiconductor Proc. 2017. 64. P. 1015 (http://dx.doi.org/10.1016/j. mssp.2017.02.026).

7. Schroeder J., Rosolowski J.H. Light scattering in polycrystalline materials. Proc. SPIE. 1982. 297. P. 156-168 (doi: 10.1117/12.932497). 
8. Adams J.H. Specifications for optical grade germanium and silicon blanks. Proc. SPIE. 1983. 406. P. 51-60 (doi: 10.1117/12.935669).

9. Van Goethem L., Van Maele L.Ph., Van Sande M., Trade-offs using poly versus monocrystalline Germanium for infrared optics. Proc. SPIE. 1986. 683. P. 160-165 (doi: 10.1117/12.936432).

10. Kaplunov I.A., Kolesnikov A.I., Talyzin I.V., Shaiovich S.L. Light scattering by single crystals of paratellurite and germanium. J. Opt. Technol. 2005. 72, No 3. P. 271-275 (doi: 10.1364/JOT.72.000271).

11. Hibbard D., Neff B., Klinger B., Stout M. Critical performance differences of monocrystalline versus polycrystalline germanium for optical application Light Works Optical Systems Proprietary Information, accessed at http://opticalsystems.com/files/7113/8558/1721/Ge _Mono_vs_Poly_paper-A.pdf

12. McNatt J.L., Handler P. Interband optical properties of grain boundaries in germanium: An amorphous system. Phys. Rev. 1969. 178. P. 1328-1336.

13. Lewis C.L., Runalls R.H., Turner G.N., Davies S.T. Optical requirements for thermal imaging lenses. Proc. SPIE. 1979. 163. P. 1-7.

14. Pekar G.S., Singaevsky A.F. Improved optical germanium: unconventional dopants, self-controlled technology. Materials of the E-MRS IUMRS ICEM 2006 Spring Meeting, Nice, France, 2006, P. 2-07.

15. Claeys C., Simoen E. Germanium-Based Technologies - From Materials to Devices. Elsevier, Amsterdam, 2007, and references therein.

16. Kaplunov I., Kolesnikov A. Effect of Germanium characteristics on IR radiation scattering surface. $X$-ray, Synchrotron and Neutron Investigations. 2002. 2. P. 14-19 (in Russian).

17. Kalinushkin V.P., Voronkov V.V., Voronkova G.I., Golovina V.N., Zubov B.V., Murina T.M., A.M. Prokhorov Impurity Clouds in Silicon and Germanium, In: Physical Processes in LaserMaterials Interactions, ed. M. Bertolotti. Springer Science \& Business Media, 2013, P. 405-412 (Proc. of the 1980 NATO Advanced Study Institutes Series).

18. Bagdasarov Kh.S. High-temperature Crystallization from Melt. Moscow: Fizmatlit, 2004 (in Russian).

19. Asatryan D.G. Image blur estimation using gradient field analysis. Computer Optics. 2017. 41, No 6. P. 957-962.

\section{Authors and CV}

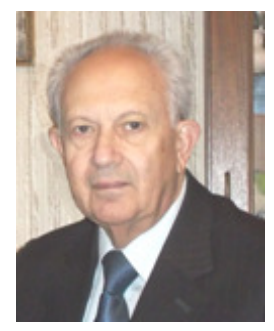

Grygoriy S. Pekar graduated from the Physical Faculty of Taras Shevchenko Kyiv State University in 1965, he works at the V. Lashkaryov Institute of Semiconductor Physics NAS of Ukraine since 1966. Received $\mathrm{PhD}$ degree in 1972 and doctoral degree in 1993 from this Institute. Professor since 1996, Head of the Department since 2009. He is the author of 170 scientific publications and patents in the field of crystal growth and physical investigations of semiconductors, mainly II-VI compounds and germanium. He was awarded with the 2010 State Prize of Ukraine in Science and Technology.

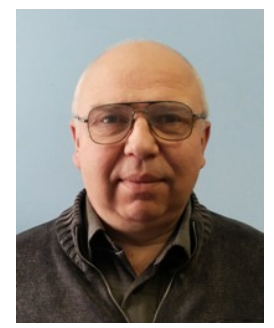

Alexander F. Singaevsky graduated from the Radiophysical Faculty of Taras Shevchenko Kyiv State University in 1984, he is working at the V. Lashkaryov Institute of Semiconductor Physics, NAS of Ukraine, since 1989. Received his $\mathrm{PhD}$ degree in 1996 from this Institute, a scientific title of Senior Research Fellow in 2003. He is the author of about 60 scientific publications and patents in the field of crystal growth and physical investigations of germanium and II-VI compounds. He was awarded with the 2010 State Prize of Ukraine in Science and Technology.

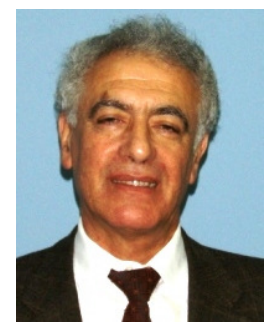

Mikhail M. Lokshin graduated from the Moscow Institute of Electronic Technology in 1973, is working at the V. Lashkaryov Institute of Semiconductor Physics, NAS of Ukraine, since 1998. He received $\mathrm{PhD}$ degree in 1984 from this Institute, a scientific title of Senior Research Fellow in 2007. He is the author of about 60 scientific publications and patents in the field of semiconductor technique and nanotechnology. He was awarded with the 2010 State Prize of Ukraine in Science and Technology. 


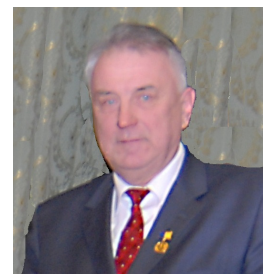

Valentin I. Gordienko graduated from the Odessa Polytechnic Institute in 1971 as radio engineer. Received $\mathrm{PhD}$ degree in 2004 and doctoral degree in 2012, both from Kyiv Polytechnic Institute. Worked at the Production Complex "Photopribor", city of Cherkasy, as General designer, Deputy General director for Science, General director. He is the author of above 150 scientific publications and patents in the field of optical instrumentation engineering. Academician of the Academy of Technological Sciences of Ukraine. He was awarded with the 2010 State Prize of Ukraine in Science and Technology.

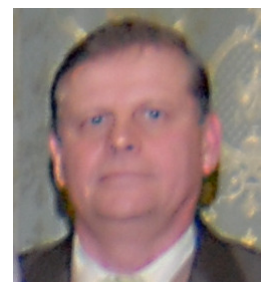

Igor V. Mazurin graduated from the Leningrad Institute of Precision Mechanics and Optics in 1983. Now he is working as Chief optician at the Research and Production Complex "Photopribor", city of Cherkasy. He is the author of 45 scientific publications and patents in the field of optical instrumentation engineering. He was awarded with the 2010 State Prize of Ukraine in Science and Technology. 